\title{
Toward Greater Preparedness and Resilience in Planetary Defense
}

\author{
Daniel R. Adamo \\ 503-585-0025 \\ Independent Astrodynamics Consultant \\ adamod@earthlink.net
}

Multiple planetary defense experts have reviewed this paper, and their substantive feedback is reflected in its content. Due to the political and possibly career-limiting nature of the paper's subject matter, however, this feedback is being made anonymously. The author is nevertheless grateful for these contributions to this paper's integrity. 


\section{Toward Greater Preparedness and Resilience in Planetary Defense}

\section{Introduction}

The most likely celestial impact threats to Earth are posed by near-Earth objects (NEOs), small bodies capable of approaching the Sun to within 1.3 times Earth's heliocentric distance, or 1.3 au [1, p. 1, Footnote 1]. Although widespread destruction from NEO impacts on Earth is a remote possibility, casualties and property damage from such an event can be cataclysmic [1, Figure 2.4]. In response to this threat, "NASA established the Planetary Defense Coordination Office [PDCO] in January 2016 to coordinate planetary defense efforts across NASA, with other US agencies, and with international partners, and to administer NASA's Near-Earth Object Observation Program [NEOOP] which dates back to 1998" [2, p. 4]. The PDCO operates within the Planetary Science Division of the Science Mission Directorate (SMD) at NASA [2, p. 3].

As illustrated by Figure 1, the vast majority of NEO discoveries are attributable to NEOOPfunded Earth-based telescope programs including the LINEAR project, the NEAT project, Spacewatch, LONEOS, the Catalina Sky Survey, Pan-STARRS1 [3, last paragraph], and ATLAS [4]. The only space-based NEO observing program is NEOWISE in Sun-synchronous low-Earth orbit (LEO), also funded by NEOOP [5].

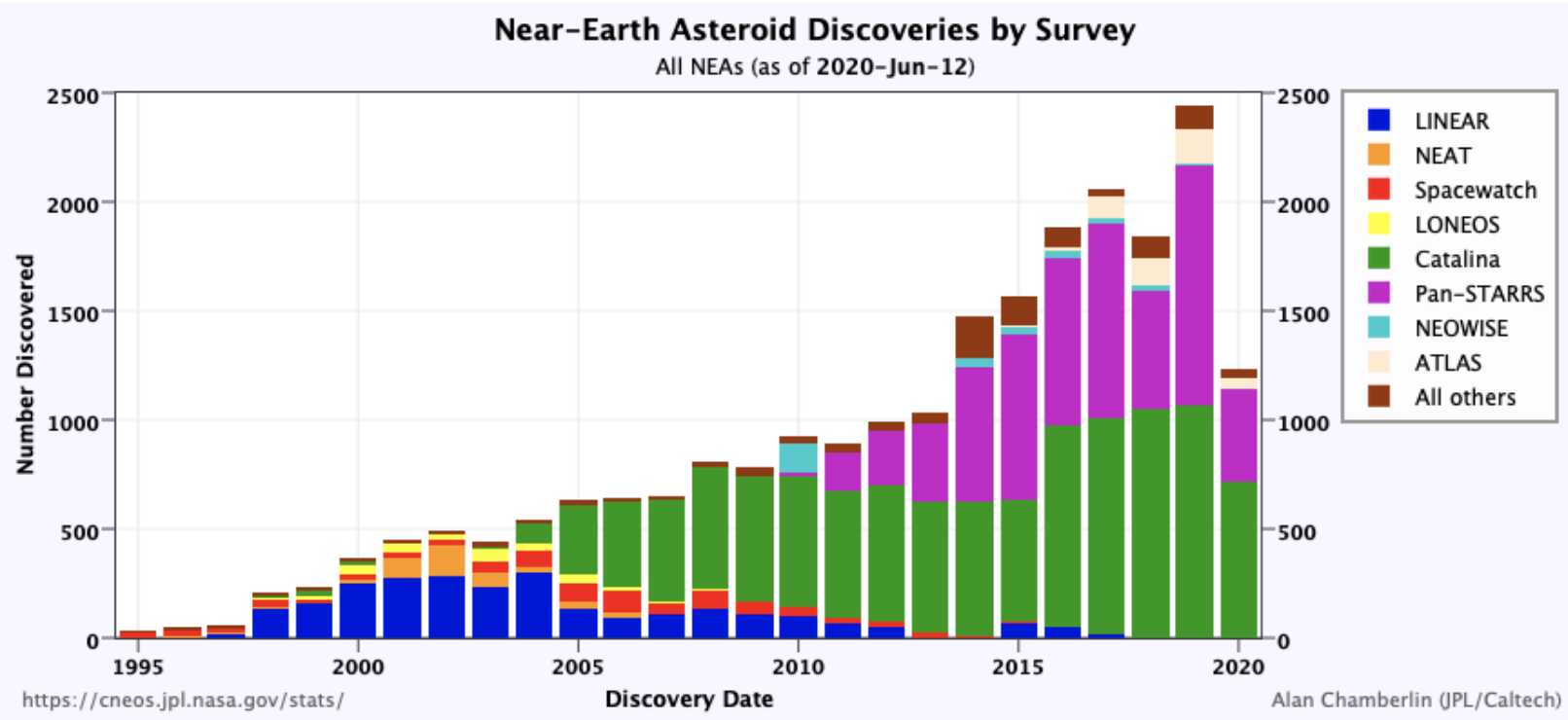

Figure 1. Yearly NEO discoveries are attributed to their respective observing programs, almost all of which are funded by NEOOP [6]. ${ }^{1}$

Funding provided by NEOOP is critical to PDCO's mission because NEO observations are Earth's first line of defense from impact hazards. All Earth impact mitigation techniques depend on these observations, and those techniques exerting the best control over averting disaster require the longest possible lead time between a hazardous NEO's identification and impact [1, Figure 5.5]. This precept leads to a mantra: "The three most important goals for planetary

\footnotetext{
${ }^{1}$ Throughout this paper, external references to "asteroid", as in Figure 1's title, should be regarded as synonymous with the more agnostic term "object". A small, inert, rocky body is connoted with "asteroid", but these objects may in fact be actively shedding debris and may contain volatile substances to a degree undetectable without close inspection. The term "NEO" therefore encompasses inert asteroids and active comets alike.
} 


\section{Toward Greater Preparedness and Resilience in Planetary Defense}

defense from near-Earth objects is [sic] to find them early, find them early, and...find them early" [7, p. 139].

A potentially hazardous object (PHO) is defined as any NEO whose heliocentric orbit is less than 7.5 million kilometers from Earth's and whose absolute magnitude $H$ (computed brightness at 1.0 au from the Earth and Sun) is less than +22 . Assuming a typical NEO albedo (the fraction of incident light being reflected) of $14 \%, H=+22$ is equivalent to a diameter of 140 meters [8]. As of 12 June 2020, the 23,063 known NEOs included 2091 PHOs, 9\% of the population [9].

The process of identifying PHOs that do and do not pose a credible threat to Earth can stretch over many years. For example, consider $2006 \mathrm{QV}_{89} .{ }^{2}$ Per its designation, this NEO was discovered in 2006 as it approached Earth to within 3.1 million kilometers on 5 September [10, click "show close-approach data"]. Post-discovery observations indicated a close Earth approach in 2019, but the uncertainty in this prediction was unable to rule out an Earth impact on 9 September 2019 because the observation arc extended over a very small fraction of the postdiscovery orbit [11, "2019-Jul-18" entry]. Only on 4/5 July 2019 was disaster discounted by European Southern Observatory Very Large Telescope observations failing to detect 2006 $\mathrm{QV}_{89}$ 's approach from the direction necessary for Earth impact. Subsequent telescopic observations recovered $2006 \mathrm{QV}_{89}$ in July and August of 2019 and ultimately refined predicted Earth miss distance to 6.9 million kilometers on 27 September [12].

Post-recovery observations of $2006 \mathrm{QV}_{89}$ in 2019 also permitted planetary radar to be pointed precisely enough to obtain an echo from this NEO during August [13]. Up to that point, all 2006 $\mathrm{QV}_{89}$ observations had been pairs of angles locating it on the terrestrial sky, and the third radial dimension of position had to be inferred statistically. Radar observations contributed range and radial velocity measurements for the first time, greatly reducing prediction uncertainty. Thus, the predicted $2006 \mathrm{QV}_{89}$ Earth approach to within 3.1 million kilometers on 19 December 2032 has miniscule uncertainty and no credible impact scenario [10, click "show close-approach data"].

In addition to precise orbit determination, planetary radar observations are able to provide NEO characterization data of relevance to Earth impact mitigation. These data include, size, topography, spin state, roughness, composition, and the presence of sufficiently prominent companion objects (moons) [1, pp. 52-53]. Companion revolution measurements also permit NEO mass and density to be estimated. Mass is the most important NEO characterization parameter relating to any Earth impact mitigation technique under consideration [1, p. 4].

Since PDCO's formation, it has bravely waged an uphill bureaucratic battle for adequate NEOOP funding. Empirical incidents, such as the foregoing $2006 \mathrm{QV}_{89}$ example, have bolstered that campaign. This paper examines the criticality of winning NEOOP's funding battle and why SMD may not be the best organizational venue from which to wage it.

\footnotetext{
${ }^{2}$ With a diameter of approximately 30 meters, this NEO is too small to be classified as a PHO. Nevertheless, its observational history could easily apply to larger objects in similar orbits.
} 


\section{Toward Greater Preparedness and Resilience in Planetary Defense}

\section{The Elusive Space-Based NEO Survey}

In its 2005 NASA Authorization Act, Congress calls for NASA to discover and physically characterize before 2021 at least $90 \%$ of NEOs whose diameters exceed 140 meters [14, Sec. 321]. Known as the George E. Brown, Jr. Near-Earth Object Survey (GEB), no funding has yet been provided by Congress for this specific purpose [15, p. 2]. Within the GEB's scope are NEOs thought to be capable of imparting at least regional damage through Earth impact [1, Table 2.1]. The GEB deadline will not be met. All NEOs with diameters exceeding 140 meters are thought to number about 25,000 [16, Figure 7 evaluated for absolute magnitude $H=+22$ ], but only 9145 had been discovered as of 10 June 2020 [9]. Thus, the GEB is about one-third complete 15 years after it became law.

It appears the current strategy to achieve deferred GEB goals involves three primary components.

1) The operational ground-based Pan-STARRS telescopes [17]

2) The ground-based Large Synoptic Survey Telescope (LSST), now known as the Vera C. Rubin Observatory (VRO), expected to be operational in 2023 [18]

3) The space-based Near-Earth Object Surveillance Mission (NEOSM) expected to be launched no earlier than 2025 [19]

After these three components are operational, the GEB 90\% requirement is estimated to be satisfied in about 10 years [15, p. 34]. Without NEOSM or similar space-based sensors, however, this milestone could be delayed indefinitely [15, pp. 26-27].

In addition to its unique perspective, about 1.5 million kilometers towards the Sun from Earth at the first Sun-Earth Lagrange point (SEL1), NEOSM will contribute critical NEO characterization data to the GEB. Because it observes at infrared wavelengths, where NEOs are self-luminous, NEOSM will be able to associate NEO diameter with observed brightness and statistically inferred distance to about $25 \%$ precision [15, Table 5.1]. With diameter and $H$ known, albedo can be determined. Spectral class constraints follow from albedo such that gross composition and mean density can be inferred. From diameter and density, a rough value for the NEO's mass is estimated.

Infrared wavelengths are inaccessible to ground-based telescopes whose reflected visible light observations cannot discriminate between large, low-albedo NEOs and proportionally small, high-albedo ones. Consequently, if plausible visible light albedos for NEOs range from 3\% to $60 \%$, corresponding diameter estimates will assume normalized values from 4.47 to 1.00 [20]. Without infrared observations, NEOs thought to be near 140 meters in diameter based on visible light observations may or may not actually be PHOs.

There is, however, little reassurance to be taken from being at "ground zero" when an impacting NEO somewhat less than 140 meters in diameter strikes Earth. The Tunguska event in 1908 destroyed over 2000 square kilometers of Siberian forest and is thought to have been caused by atmospheric explosion of an object 30 to 70 meters in diameter [1, p. 3]. In 2013, an object 17 to 20 meters in diameter moving at 18.6 kilometers per second exploded at 23 kilometers' altitude south of Chelyabinsk, Russia with an energy of 440 kilotons [21]. The resulting shock wave injured over 1600 people [15, p. 2]. Therefore, detecting 100\% of the GEB targets will provide 


\section{Toward Greater Preparedness and Resilience in Planetary Defense}

only incomplete situation awareness regarding all NEO threats to life and property on Earth. For surveillance purposes, the threshold for such threats appears to be near a diameter of 20 meters.

Nevertheless, achieving the GEB deferred goals will advance planetary defense aims while also contributing to scientific knowledge of NEOs and to supplementing the catalog of accessible destinations for human off-Earth exploration. These GEB returns on investment have motivated NASA's Small Bodies Assessment Group (SBAG) to steadfastly advocate funding a space-based survey telescope since 2011 [22, SBAG \#5 Finding 1, SBAG \#6 Finding 2, SBAG \#8 Finding 6, SBAG \#9 Finding 4, SBAG \#10 seventh finding, SBAG \#11 first and seventh findings, SBAG \#12 first finding, SBAG \#13 fifth finding, SBAG \#16 second finding, SBAG \#17 fourth and fifth findings, SBAG \#19 second finding, SBAG \#20 tenth finding, SBAG \#21 third finding, and SBAG \#22 first finding].

Despite SBAG's advocacy, little progress over the past decade has been made toward actually deploying a space-based survey telescope. Although PDCO has funded an extended Phase A study to develop such a telescope, anything like a commitment to launch a space-based NEO survey mission did not occur until September 2019, when NEOSM was first defined. Due to PDCO's limited budget, a firm commitment to launch NEOSM must await completion of the higher priority Double Asteroid Redirection Test (DART), due for launch in 2021. As a reality check, consider NEOSM's estimated \$500-600 million cost. Given PDCO's \$150 million annual budget [23], funding NEOSM is a sobering long-term commitment.

A space-based NEO survey capability is largely justified by the GEB, which is unfortunately a classic example of the unfunded Congressional mandate. Compounding this handicap is the 2016 decision [2, p. 4] to establish the PDCO (a largely operations-driven organization) within SMD (a largely science-driven organization). This organizational conflict of interest appears to have led Dr. Thomas Zurbuchen, SMD Associate Administrator since 2016 [24], to call delayed funding for a space-based NEO survey "one of the biggest screwups I've made in my job" [25]. An additional motivation for Dr. Zurbuchen's reordered priorities may have been the discovery of NEO 2019 OK, estimated to be from 60 to $130 \mathrm{~m}$ in diameter, one day before it passed 65,000 kilometers from Earth's surface on 25 July 2019 Universal Time [26]. This eleventh-hour discovery demonstrates the limitations of observations confined to Earth's surface and LEO.

\section{The Truant Planetary Radar}

The unique role planetary radars play in planetary defense has been documented in Section 1. According to the Jet Propulsion Laboratory, "Radar is the most powerful astronomical technique for characterizing near-Earth objects and refining their orbits" [27]. Only two facilities capable of transmitting an outbound planetary radar pulse exist, and neither is dedicated to function in this manner. ${ }^{3}$ Two radar-transmitting antennae at Goldstone, California are usually communications elements of NASA's Deep Space Network, and the antenna at Arecibo, Puerto Rico usually serves as a radio telescope [1, p. 3]. These time-sharing arrangements function remarkably well, even in response to unforeseen observing opportunities, but other obligations can frequently leave a planetary radar unavailable for weeks at a stretch.

\footnotetext{
${ }^{3}$ Additional radio telescope facilities are capable of passively detecting the returning radar pulse, but these are not dedicated to this function either.
} 


\section{Toward Greater Preparedness and Resilience in Planetary Defense}

Both planned and unforeseen events all too often arise to disable either or both planetary radar facilities. Since 2010, SBAG findings have persistently recognized the value of planetary radar observations, together with the vulnerability of associated infrastructure to critical parts failures and natural disasters as compounded by slim funding margins for recovery [22, SBAG \#2 Finding 2, SBAG \#8 Finding 5, SBAG \#11 fifth finding, SBAG \#14 fifth finding, SBAG \#15 sixth finding, SBAG \#16 fourth finding, SBAG \#17 third finding, SBAG \#18 sixth finding, SBAG \#19 eighth finding, SBAG \#20 sixth finding, and SBAG \#22 fifth finding], [28, SBAG \#23 Finding 4]. The signal sources for both planetary radars are klystrons sole-sourced from Communications \& Power Industries (CPI, formerly Varian). Funding is inadequate to maintain a stockpile of klystrons, so a planetary radar may be degraded or inoperative for a year or two when a klystron must be replaced. Arecibo was unavailable for three months following Hurricane Maria damage in 2017 and was inoperative again for a month due to earthquakes in January 2020. Planned maintenance for Arecibo typically renders it unavailable for 2-week intervals, while planned maintenance for Goldstone usually extends over several months.

During March and April of 2020, no planetary radar capability was available for multiple weeks. At the time, Goldstone was awaiting a klystron replacement not expected to be completed until that summer. Meanwhile, Arecibo was closed while a COVID-19 social distancing policy for its personnel was approved by the Puerto Rican government [29, 1 June 2020 presentation by Kelly Fast]. Whenever a total planetary radar outage arises, the NEOOP mission is significantly compromised. Because these facilities lack two-fault-tolerant redundancy, a fundamental precept in critical systems design, the likelihood of total outages could be significantly reduced by commissioning and maintaining a third planetary radar at a well-distanced location, ideally offering improved access to the southern sky. All planetary radar installations should have access to a robust stockpile of critical parts, receive upgrades extending the range at which NEOs larger than 20-meter diameter can be observed, and possess a government-approved disaster plan.

Preserving the planetary radar status quo is likely to dramatically increase the number of newly discovered NEOs whose Earth collision prospects cannot be totally discounted. Simulated operations over ten years indicate VRO discoveries will include about half of the 25,000 NEOs whose diameters exceed 140 meters [16, p. 45]. In other simulations, NEOSM is projected to discover two-thirds of these NEOs in only four years [30]. Still more prolific discoveries by these instruments will involve smaller NEOs only observable during a close Earth encounter. Many of these NEOs will pose an Earth impact threat that can only be immediately eliminated by planetary radar observations carried out on short notice. Without such observations, potential impact threats can persist for years, as noted in Section 1.

\section{Conclusion}

Imagine a nation's military defense with early warning systems suffering from inadequate territorial coverage and extended outages. Surprise attacks on such a defense would be easy to plan and execute, inflicting grievous harm on the nation. In the planetary defense analog to these hypothetical warning systems, humanity can only be grateful NEOs and other natural Earth impact threats are not the result of strategic planning by a hostile power. The "find them early" mantra in planetary defense demands continual preparedness and resilience from the NEOOP. 


\section{Toward Greater Preparedness and Resilience in Planetary Defense}

Although technology enabled this degree of readiness more than a decade ago, SMD's budget for NEOOP has not.

To further compound NEOOP's funding shortfall, its aims are currently in competition with DART mission costs. Returning to the military defense analog, it is as though an antimissile system is being tested before a means to reliably detect and target enemy missiles is fully operational. Both NEOSM and DART are worthwhile missions, but surveillance measures like NEOSM should have priority over mitigation tests like DART in the absence of future funding sufficient to develop and operate both in parallel.

Switching to a medical analog, responsive/redundant/resilient planetary radar capability for Earth's defense is akin to an adequate supply of infection/immunity tests during the COVID-19 pandemic. Without these situation awareness prerequisites, mitigating the threats they would identify is problematic at best.

Surveilling and characterizing NEOs resembles the Global Positioning System at its inception in at least one respect: everyone wants it, but nobody wants to pay for it. Clearly, the NEOOP funding shortfall and associated priority conflicts cry out for a NASA-independent assessment. Requirements to ensure NEOSM launch without significant delay beyond 2025 should be identified, together with what it will take to maintain a planetary radar capability responsive to both predicted and unanticipated future Earth encounters by all observable NEOs with diameters larger than 20 meters. If resources to satisfy these requirements cannot be provided through $\mathrm{SMD}$, recommendations for moving the PDCO to an organization more aligned with its operations-focused defense mission are appropriate. Although the report from this assessment should be distributed without restriction, its primary stakeholders are likely in the U.S. Congress and NASA. It is hoped this report will greatly advance progress toward the ideal of "Finding Them Before They Find Us" as expressed in the subtitle of an authoritative book on defense from NEO impacts [7].

\section{References}

[1] NRC Committee to Review Near-Earth Object Surveys and Hazard Mitigation Strategies, Defending Planet Earth: Near-Earth Object Surveys and Hazard Mitigation Strategies:

Final Report, National Academies Press, 2010. ${ }^{4}$

[2] Planetary Defense Coordination Office, Planetary Defense at NASA Press Kit, NASA, 2019. 5

[3] https://cneos.jpl.nasa.gov/about/search_program.html (accessed 12 June 2020).

[4] https://fallingstar.com/nasa_funding.php (accessed 12 June 2020).

[5] https://neowise.ipac.caltech.edu (accessed 12 June 2020).

[6] https://cneos.jpl.nasa.gov/stats/site_all.html (accessed 12 June 2020).

[7] D. K. Yeomans, Near-Earth Objects: Finding Them Before They Find Us, Princeton University Press, 2013.

[8] https://cneos.jpl.nasa.gov/about/neo_groups.html (accessed 13 June 2020).

\footnotetext{
${ }^{4}$ This document may be downloaded at http://www.nap.edu/catalog/12842.html (accessed 12 June 2020).

${ }^{5}$ This document may be downloaded at https:/www.nasa.gov/sites/default/files/atoms/files/pdco-press-kit04232019-v3.pdf (accessed 12 June 2020).
} 


\section{Toward Greater Preparedness and Resilience in Planetary Defense}

[9] https://cneos.jpl.nasa.gov/stats/totals.html (accessed 13 June 2020).

[10] https://ssd.jpl.nasa.gov/sbdb.cgi?sstr $=2006 \% 20 \mathrm{QV} 89 ;$ old $=0 ; \mathrm{orb}=0 ; \mathrm{cov}=0 ; \log =0 ; \mathrm{cad}=1 \#$ cad $(\operatorname{accessed} 13$ June 2020).

[11] https://cneos.jpl.nasa.gov/sentry/notes.html (accessed 13 June 2020).

[12] https://cneos.jpl.nasa.gov/news/news204.html (accessed 13 June 2020).

[13] https://echo.jpl.nasa.gov/ lance/radar.nea.periods.html (accessed 13 June 2020).

[14] 109th Congress, Public Law 109-155, $2005 .{ }^{6}$

[15] Committee on Near Earth Object Observations in the Infrared and Visible Wavelengths, Finding Hazardous Asteroids Using Infrared and Visible Wavelength Telescopes, National Academies Press, 2019. ${ }^{7}$

[16] S. R. Chesley and P. Vereš, " Projected Near-Earth Object Discovery Performance of the Large Synoptic Survey Telescope", CNEOS, JPL 16-11, 2017. ${ }^{8}$

[17] https://www.ifa.hawaii.edu/research/Pan-STARRS.shtml (accessed 15 June 2020).

[18] https://www.lsst.org/about/timeline (accessed 15 June 2020).

[19] https://uanews.arizona.edu/story/uarizona-looks-toward-work-nasa-s-potentialasteroidhunting-space-telescope (accessed 15 June 2020).

[20] https://cneos.jpl.nasa.gov/tools/ast_size_est.html (accessed 15 June 2020).

[21] https://www.jpl.nasa.gov/news/news.php?release=2013-061 (accessed 16 June 2020).

[22] https://www.lpi.usra.edu/sbag/findings/archive/ (accessed 29 June 2020).

[23] https://spacenews.com/nasa-to-develop-mission-to-search-for-near-earth-asteroids/ (accessed 17 June 2020).

[24] https://www.nasa.gov/feature/thomas-zurbuchen-associate-administrator-for-science/ (accessed 17 June 2020).

[25] https://www.space.com/nasa-to-build-near-earth-asteroid-hunter-telescope.html (accessed 17 June 2020).

[26] https://cneos.jpl.nasa.gov/news/news203.html (accessed 29 June 2020).

[27] https://echo.jpl.nasa.gov/ lance/nea_radar.pdf (accessed 19 June 2020).

[28] https://www.lpi.usra.edu/sbag/meetings/jun2020/Findings_23_final_June_20_2020.pdf (accessed 29 June 2020).

[29] https://www.lpi.usra.edu/sbag/meetings/jun2020/ (accessed 19 June 2020).

[30] https://neocam.ipac.caltech.edu/page/mission (accessed 20 June 2020).

\footnotetext{
${ }^{6}$ This document may be downloaded at https://www.congress.gov/109/plaws/publ155/PLAW-109publ155.pdf (accessed 15 June 2020).

${ }^{7}$ This document may be downloaded at https://www.nap.edu/download/25476 (accessed 13 July 2020).

8 This document may be downloaded from https://cneos.jpl.nasa.gov/doc/JPL_Pub_16-11_LSST_NEO.pdf (accessed 20 June 2020).
} 